\title{
Elderly patients with ESRD at risk of functional decline after initiation of dialysis
}

E lderly nursing home residents with end-stage renal disease (ESRD) who undergo dialysis can experience a sizeable and sustained deterioration in their ability to carry out basic daily functions, according to a study published in the New England Journal of Medicine. "One year after starting dialysis, only one in eight nursing home patients had survived and maintained their functional status at predialysis levels," reports lead investigator Manjula Kurella Tamura, of Stanford University School of Medicine.

Elderly patients represent the fastest growing segment of the ESRD population; in the US, nursing home residents account for around $11 \%$ of new ESRD cases in the US who are over the age of 70 years. The number of such patients starting dialysis seems to have increased over the past decade. However, the benefits of dialysis in elderly patients are unclear. Specifically, whether dialysis extends the life span of the sickest patients in this group, such as those who have multiple chronic diseases other than kidney disease, is unclear. Kurella Tamura explains that "one rationale for starting dialysis in individuals in the sickest patient group is that the treatment may improve functioning and/or alleviate symptoms, even if it does not extend life." The investigators therefore sought to determine whether dialysis does improve the functional status of elderly patients.

Kurella Tamura et al. retrospectively analyzed data from the US Renal Data System database for 3,702 nursing home residents with ESRD (mean age 73.4 years) who started dialysis between June 1998 and October 2000. Functional status, which has an important bearing on quality of life, was assessed before and after the initiation of dialysis by means of the Minimum Data Set-Activities of Daily Living (MDS-ADL) score. The MDS-ADL score uses a scale of 0-28 and measures the ability of an individual

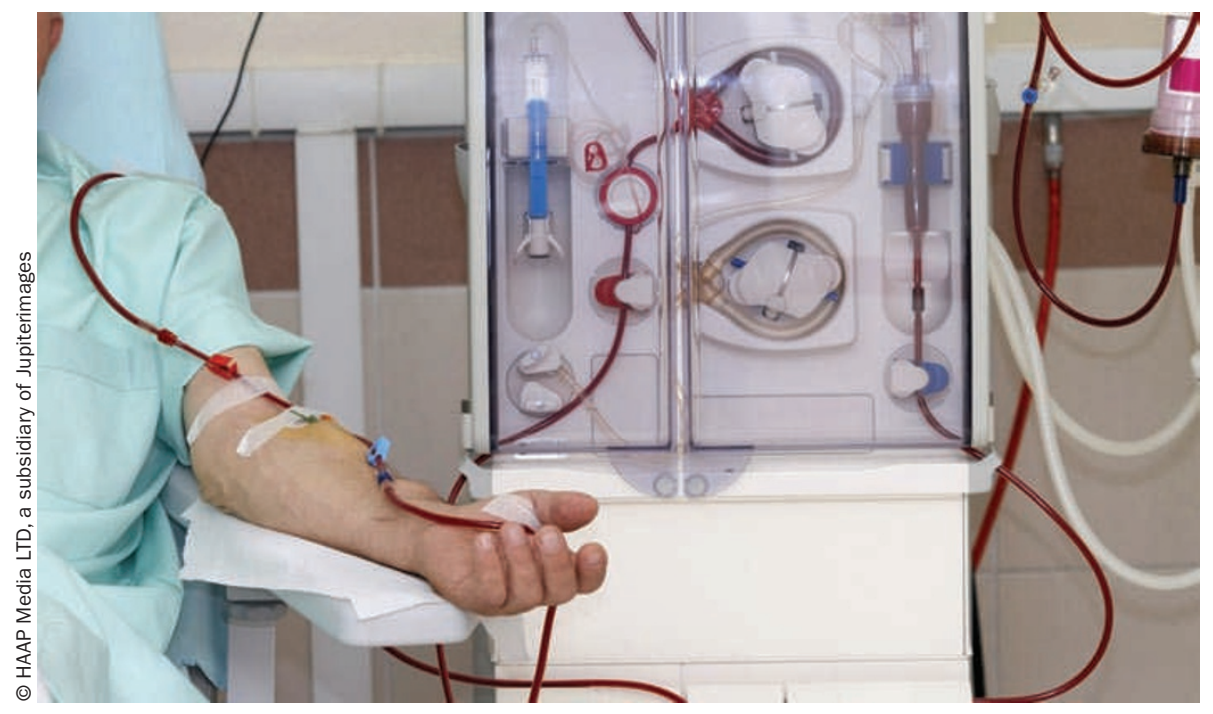

to perform activities that are considered essential for independence, such as walking, maintaining personal hygiene and moving from a seated to standing position. A high score represents increased difficulty in performing these activities.

In the current study, mortality was $24 \% 3$ months after initiation of dialysis, and increased to $58 \%$ after 12 months. The median MDS-ADL score increased from 12 in the 3 months before dialysis to 163 months after dialysis was started. "For most patients, this disability was permanent, that is, dialysis did not help patients maintain their predialysis level of functioning," Kurella Tamura comments. Functional status before dialysis was only maintained in $13 \%$ of those who survived. The decline in functional status after the initiation of dialysis was independent of race, age, sex or changes in functional status before the initiation of dialysis.

As Kurella Tamura and colleagues describe in their paper, several factors could have contributed to the observed decline in functional status, such as comorbidities and/or disabilities, hospitalization or events leading to hospitalization, the physical risks associated with dialysis, or multiple organ failure of which kidney failure was indicative. Functional decline was greatest in the oldest individuals and in patients who were hospitalized.

The researchers believe that their results provide useful information about the potential outcomes of dialysis in elderly patients with ESRD. They say that this information can assist the decisionmaking process with regard to the medical care of such patients.

Kurella Tamura suggests that future research should address whether alternative approaches to management of chronic kidney disease prevents disability in elderly patients. "This approach might include implementing a geriatric rehabilitation program before patients develop ESRD, or a trial of delayed initiation of dialysis." She adds that the present findings also indicate that palliative care could be an appropriate management strategy for some of elderly patients with ESRD.

\section{Lisa Richards}

Original article Kurella Tamura, M. et al. Functional status of elderly adults before and after initiation of dialysis. N. Engl. J. Med. 361, 1539-1547 (2009) 